\title{
A incidência de tromboembolismo venoso em gestantes e no puerpério e seus fatores de risco
}

\author{
The incidence of venous thromboembolism in pregnant and postpartum women and their \\ risk factors
}

\author{
La incidencia de tromboembolismo venoso en mujeres embarazadas y posparto y sus \\ factores de riesgo
}

Maysa Maria Veiga Mendonça ${ }^{1 *}$, Ana Carolina de Freitas Santos ${ }^{2}$, Anne Caroline Rogienfisz Mendes $^{3}$, Bruna Azedo Guimarães ${ }^{4}$, Giselle Assayag Ribeiro ${ }^{5}$, Isabela Abud de Andrade ${ }^{4}$, Leticia Stefanelli Potsch ${ }^{6}$, Lorena Souza de Jesus ${ }^{4}$, Luiza Fernanda Mendonça Nicolau ${ }^{4}$, Maria Luísa Barros Quintão Couto Parreira ${ }^{7}$.

\section{RESUMO}

Objetivo: Esse artigo buscou discutir a incidência do Tromboembolismo Venoso (TEV) em mulheres durante a gravidez e o puerpério. Além disso, também foram discutidos os principais fatores de risco associados e a melhor conduta para essa complicação, que é uma das principais causas de mortes evitáveis no período de puerpério em ambiente intra-hospitalar. Revisão bibliográfica: $O$ trabalho trata-se de uma revisão bibliográfica narrativa, em que foi abordado a incidência, a fisiopatologia e os fatores de riscos pré-existentes para TEV, como idade, tabagismo, obesidade, diabetes, além de fatores obstétricos, a saber, gestações múltiplas, pré-eclâmpsia, cesariana, infecções e hemorragias pós-parto. Na conduta, a tromboprofilaxia deve ser realizada após estratificar o risco de cada paciente, e em casos de intermediário e alto risco deve-se realizar terapia medicamentosa com uso de anticoagulantes. Considerações finais: Por ser uma doença muito recorrente, é necessária a estratificação de riscos para TEV desde o primeiro trimestre de gestação, além do acompanhamento profissional durante o ciclo gravídico-puerperal, visto que o diagnóstico precoce é muito importante para o melhor prognóstico.

Palavras-chave: Complicações na gravidez, Período pós-parto, Tromboembolia venosa.

\begin{abstract}
Objective: This article sought to discuss the incidence of Venous Thromboembolism (VTE) in women during pregnancy and the puerperium. In addition, the main associated risk factors and the best course of action for this complication, which is one of the main causes of preventable deaths in the puerperium period in an inhospital environment, were also discussed. Bibliographic review: The work is a narrative bibliographic review, in which the incidence, pathophysiology and pre-existing risk factors for VTE, such as age, smoking, obesity, diabetes, as well as obstetric factors, such as multiple pregnancies, pre -eclampsia, cesarean section, infections and postpartum hemorrhages. In the management, thromboprophylaxis must be performed after stratifying the risk of each patient, and in cases of intermediate and high risk, drug therapy with the use of anticoagulants should be performed. Final considerations: As it is a very recurrent disease, it is necessary to stratify risks for VTE since the first trimester of pregnancy, in addition to professional monitoring during the pregnancy-puerperal cycle, since early diagnosis is very important for the best prognosis.
\end{abstract}

Key words: Postpartum period, Pregnancy complications, Venous thromboembolism.

\footnotetext{
${ }^{1}$ Pontifícia Universidade Católica de São Paulo (PUCSP), São Paulo - SP.

*E-mail: maysa.veiga2@gmail.com

2 Universidade Federal do Pará (UFPA), Belém - PA.

3 Universidade Fundação Oswaldo Aranha (UniFOA), Volta Redonda - RJ.

${ }^{4}$ Universidade Nilton Lins (UNL), Manaus - AM.

${ }^{5}$ Universidade Federal do Amazonas (UFAM), Manaus - AM.

${ }^{6}$ Universidade Vila Velha (UVV), Vila Velha - ES.

${ }^{7}$ Faculdade de Medicina do Vale do Aço (FAMEVAÇO), Ipatinga - MG.
} 


\section{RESUMEN}

Objetivo: Este artículo buscó discutir la incidencia de Tromboembolismo Venoso (TEV) en mujeres durante el embarazo y el puerperio. Además, también se discutieron los principales factores de riesgo asociados y la mejor línea de actuación para esta complicación, que es una de las principales causas de muerte prevenible en el puerperio en un ambiente intrahospitalario. Revisión bibliográfica: El trabajo es una revisión bibliográfica narrativa, en la que se analiza la incidencia, fisiopatología y factores de riesgo preexistentes de TEV, como edad, tabaquismo, obesidad, diabetes, así como factores obstétricos, como embarazos múltiples, preeclampsia, cesárea, infecciones y hemorragias posparto. En el manejo se debe realizar tromboprofilaxis luego de estratificar el riesgo de cada paciente, y en los casos de riesgo intermedio y alto se debe realizar terapia farmacológica con el uso de anticoagulantes. Consideraciones finales: Al tratarse de una enfermedad muy recurrente, es necesario estratificar los riesgos de TEV desde el primer trimestre del embarazo, además del seguimiento profesional durante el ciclo embarazo-puerperal, ya que el diagnóstico precoz es muy importante para el mejor pronóstico.

Palabras clave: Complicaciones del embarazo, Periodo posparto, Tromboembolia venosa.

\section{INTRODUÇÃO}

O tromboembolismo venoso é uma das principais causas de mortes evitáveis no período de puerpério em ambiente intra-hospitalar. Essa condição é caracterizada pela ocorrência de duas alterações: a Trombose Venosa Profunda (TVP), que ocorre no pulmão, e a Tromboembolia Pulmonar (TEP), sem causa idiopática ou conhecida, geralmente nos membros inferiores (SILVA IGL, et al., 2019).

O risco desta complicação está associado às mudanças homeostáticas que ocorrem durante este período. Assim, o aumento das concentrações de coagulação, fatores VII, VIII, IX, X e XII, fibrinogênio, fator de von Willebrand, resulta em um estado hipercoagulável, levando, consequentemente, essas mulheres à um risco elevado de trombose (MOHAMMED AA, et al., 2020). A título de exemplificação, a prevalência de TVP é de 0,5 a 2,2 casos para cada 1000 partos (OLIVEIRA ALML, et al., 2020).

A gestante apresenta os sinais que caracterizam a Tríade de Virchow, que corresponde a três categorias de fatores que contribuem para a TVP, a saber, a estase venosa, devido à compressão das veias pelo útero gravídico, a hipercoagulabilidade, pela alteração de fatores como o fibrinogênio, e a lesão endotelial, presente na nidação, na remodelação endovascular das artérias uteroespiraladas e na dequitação. Além disso, a TVP é mais comum no membro inferior esquerdo, geralmente, devido à intensa compressão da veia ilíaca comum esquerda pela artéria ilíaca comum direita contra a quinta vértebra lombar, gerada pelo útero gravídico (SOUZA EO, et al., 2021; OLIVEIRA ALML, et al., 2020).

Os principais Fatores de Risco (FR) para esses fenômenos de tromboembolia venosa profunda na gestação e no puerpério são idade maior que 30 ou 35 anos, multiparidade, gemelaridade, longa permanência no leito, cesariana, hipertensão específica da gestação, diabetes gestacional, obesidade, hiperêmese gravídica, história pessoal ou familiar de trombose, hemorragia pós-parto, anemia falciforme, lúpus eritematoso sistêmico, infecções ou internações na gravidez, trabalho de parto prematuro, trombofilia e tabagismo. Além disso, percebe-se que identificar esses FR para TVP nessas mulheres facilita o diagnóstico ou a realização de profilaxia (HILLMANN BR, et al., 2015).

Considerando a relevância do tema abordado, a presente revisão tem como objetivo descrever a incidência do tromboembolismo venoso em mulheres durante a gestação e no período puerperal e seus principais fatores de risco, assim como abordar os melhores protocolos para que tal complicação seja evitada e tratada corretamente.

\section{REVISÃO BIBLIOGRÁFICA}

O tromboembolismo venoso é caracterizado por tromboembolia pulmonar e trombose venosa profunda, principalmente de membros inferiores. Nota-se, também, que é uma das principais causas de morbimortalidade materna no mundo ocidental durante a gravidez e o puerpério, principalmente, pelo estado 
de hipercoagulação que o organismo feminino se encontra durante este período (EVANGELISTA MS, et al., 2018). A maioria dos estudos realizados afirma que o risco de Tromboembolismo Venoso (TEV) é maior no puerpério que na gravidez, em especial na primeira semana pós-parto, em que esse risco se torna 37 vezes maior (GALAMBOSI PJ, et al., 2017). Além disso, os locais mais comuns em que ocorre a TVP na gestação são nas veias iliofemorais. Dessa forma, o risco de desenvolver embolia pulmonar é maior quando a TVP acontece na via proximal (HILLMANN BR, et al., 2015).

A tríade de Virchow é composta pelo estado de hipercoagulabilidade, estase venosa e lesão endotelial, que consiste na principal causa de TEV durante a gravidez e o puerpério. A hipercoagulação é uma adaptação do organismo materno às demandas hemostáticas, com intuito de protegê-lo de sangramento excessivo durante o parto. Com isso, desde a concepção, iniciam-se as alterações na cascata de coagulação, como a diminuição das proteínas anticoagulantes C e S e o aumento de fibrinogênio e dos fatores V, VIII, IX e X. A estase venosa nas extremidades inferiores ocorre pela compressão uterina, juntamente com a vasodilatação induzida pela progesterona. Por fim, a lesão endotelial acontece devido ao parto, principalmente, em complicações que resultam em um trauma cirúrgico (UNGER HW, et al., 2018).

O ciclo gravídico-puerperal é um fator de risco importante para TEV, que é uma causa significativa de morbimortalidade obstétrica, representando a segunda causa de mortalidade materna no mundo. (OLIVEIRA ALML, et al., 2020). A título de exemplificação, no Reino Unido, entre 2009 e 2013, constatou-se 64 casos de mortes maternas registradas por TEV; no Japão, entre 2010 e 2012, ocorreram 14 casos de óbito materno, representando 6,5\% de todas as mortes maternas (VOON HY, et al., 2018). Nos Estados Unidos da América, estima-se que a cada 100.000 partos, a taxa de óbitos chegou a 9,2\% (ABE K, et al., 2019).

As gestantes têm o risco de apresentar TEV aumentado em até 4 vezes, quando comparadas a não gestantes na mesma faixa etária, o que pode aumentar em até 10 vezes durante o puerpério. Segundo dados epidemiológicos recentes da última década, o aumento substancial da ocorrência de TEV em gestantes ocorreu significativamente. Sabe-se que o risco para esse acometimento se inicia a partir dos primeiros 3 meses da gestação e persiste durante toda a gravidez, aumentando no terceiro trimestre, que tem taxa de internação de $17 \%$, e aumentando durante o pós-parto, com taxa de internação de $47 \%$, especialmente durante os primeiros 7 dias (SANCHES SM, et al., 2020).

Além das alterações fisiológicas associadas à gravidez no sistema sanguíneo, existem FR associados ao acometimento dessas gestantes, como a acentuada elevação na atividade pró-coagulante, por consequência da elevação dos fatores de coagulação e uma queda importante dos anticoagulantes fisiológicos. Além disso, fatores de risco pré-existentes contribuem ainda mais para condições trombóticas, tanto na gravidez quanto no puerpério (HART C, et al., 2020).

Dentre os fatores de risco pré-existentes, constata-se o histórico pessoal de tromboembolismo venoso profundo ou embolia pulmonar, sendo o risco de acometimento aumentado em até 4 vezes quando gestante, e risco absoluto quando estas não fazem profilaxia farmacológica, de 2,5\% em grávidas com histórico de apenas um episódio de TEV (HART C, et al., 2020; PANASSOL MRC, et al., 2020).

As mulheres que apresentaram o primeiro episódio de TEV relacionado à gravidez ou ao período pósparto têm um risco maior em uma gravidez subsequente, diferente das gestantes que tiveram o primeiro episódio de TEV não relacionado à gravidez. Trombofilia hereditária e histórico familiar de TEV também são FR importantes, uma vez que o histórico de trombofilia hereditária é encontrado em gestantes que apresentaram TEV no mínimo uma vez, bem como o histórico familiar de TEV, o qual está presente em um número substancial de grávidas que apresentaram a patologia (HART C, et al., 2020; PANASSOL MRC, et al., 2020).

As condutas mais importantes para o controle da mortalidade materna em relação à ocorrência de TEV são dependentes de alguns fatores, dentre eles a realização de uma avaliação de risco eficiente ainda nas primeiras semanas de gravidez, haja visto a possibilidade de ocorrência dessa alteração desde o início da gestação, podendo se estender até o puerpério (RAMOS ASMB, et al., 2018). Dessa forma, justifica-se a importância de se proporcionar uma assistência de qualidade à saúde da mulher durante o período gravídico- 
puerperal, favorecendo, assim, a prevenção de doenças indesejáveis, atentando-se aos indicadores de gravidade e obedecendo aos critérios de avaliação. Além disso, a implementação de um monitoramento periódico tem potencial destacado, já que proporciona avaliação contínua no que diz respeito à ocorrência de FR, minimizando, então, as chances de desfechos desfavoráveis (SOUZA IA, et al., 2019).

Além das alterações fisiológicas e anatômicas que caracterizam o período da gravidez, devemos considerar que existem outras condições que influenciam no surgimento de TEV. Logo, os principais fatores de risco associados ao TEV são divididos em três grupos: pré-existentes, transitórios e relacionados à gravidez (HART C, et al., 2020). Dessa forma, para que cada paciente tenha uma conduta adequada, sendo levado em conta suas individualidades, é de extrema necessidade a avaliação correta da estratificação de risco para TEV antes da gravidez, durante a gestação e no período puerperal (BATES SM, et al., 2016).

No grupo dos fatores de risco pré-existentes, encontram-se: o histórico pessoal ou familiar de TEV, idade maior que 35 anos, tabagismo antes ou durante a gravidez, índice de massa corporal (IMC) maior que 30 , trombofilia, síndrome do anticorpo fosfolipídeo, presença de varizes, principalmente nos membros inferiores, redução da mobilidade ou outras patologias como nefropatia diabética, insuficiência cardíaca, doenças inflamatórias, câncer, entre outras. Nos fatores obstétricos e transitórios, considera-se: as gestações múltiplas, utilização de técnicas de reprodução assistida, hiperêmese gravídica, pré-eclâmpsia, cesariana, trabalho de parto prolongado com duração maior do que 24 horas, infecções ou hemorragias pós-parto, procedimentos cirúrgicos durante a gravidez ou no puerpério, diminuição da mobilidade, hospitalização, entre outros (SANCHES SMV, et al., 2020; THURN L, et al., 2018).

Um dado de extrema relevância para o diagnóstico da referida enfermidade consiste no fato de que somente $50 \%$ dos casos clinicamente suspeitos de TEV realmente estão relacionados com a doença. Por isso, os sintomas isolados não a confirmam e nem a descartam, uma vez que são diversas as principais queixas dos pacientes, dentre as quais se elencam: dor, eritema, edema, aumento de temperatura, empastamento muscular, cianose e dor à palpação (PANASSOL MRC, et al., 2020).

Para o diagnóstico clínico, identifica-se a presença de alguns fatores, como edema de membros inferiores assimétrico, dor, rigidez muscular, extremidades com calor e rubor. A suspeição clínica deve ser feita mesmo nos casos oligossintomáticos e constata-se que a embolia pulmonar pode ser o primeiro sinal (SARNO MAC, et al., 2016). Para maior exatidao do diagnostico, sao feitos alguns testes durante o exame físico, tais como o sinal de Homans, podendo ser identificado por dor ou desconforto na panturrilha após dorsiflexão passiva do pé, sinal da bandeira, consistindo em menor mobilidade da panturrilha quando comparada com o outro membro, e por último o sinal de Bancroft, caracterizado por dor à palpação da panturrilha contraestrutura óssea (STUBBS MJ, et al., 2018).

Outra forma de auxílio para o diagnóstico é a utilização da tabela do Escore de Wells, que, por sua vez, tem como utilidade estimar a probabilidade pré-teste para TVP, mostrando-se útil, então, quando avaliando pacientes suspeitos de TVP. Logo, esse Escore serve como um modelo de predição clínica, tendo como base sinais, sintomas, fatores de risco e outras possibilidades de diagnóstico. O mesmo categoriza os pacientes em três grupos, sendo eles: indivíduos com baixa, moderada ou alta probabilidade de TVP (PRESTI C, et al., 2015). Os testes de D-dímero também podem ser usados para essa suspeita, mas o limiar convencional não tem especificidade para ter valor diagnóstico incremental. Por enquanto, ele é utilizado como um preditor para TVP precoce (ZHOU ZH, et al., 2018).

Os exames de imagem são considerados como padrão-ouro para diagnóstico em suspeita de TVP na gravidez (ZHOU ZH, et al., 2018). O mais utilizado deles é a ultrassonografia (USG) pois permite avaliar a compressibilidade e o fluxo local, apesar de apresentar menor precisão, principalmente, em três quadros, quais sejam: quando em veias distais, em membros superiores bem como em pacientes assintomáticos. Já a flebografia, mesmo sendo o exame padrão-ouro, não é tão rotineiro por ser bastante invasiva para o paciente. Por fim, ressalta-se a angiotomografia e a angiorressonância, em razão da sua excelência no diagnóstico de embolia pulmonar, ainda que não apresentem muito benefício comparado ao doppler nos membros inferiores (STUBBS MJ, et al., 2018). 
A gestação e o puerpério são períodos caracterizados pela hipercoagulabilidade e elevado risco de eventos tromboembólicos, decorrentes da liberação de tromboplastina pela dequitação (SARNO MAC, et al., 2016). Além disso, a imobilização por longo período de tempo durante a gravidez e as adaptações fisiológicas para prevenção de hemorragias durante o parto são fatores que contribuem para o aumento do risco de TVP, sendo ainda maior durante as seis semanas iniciais do pós-parto (CLINTON CM, et al., 2019). Dessa maneira, na primeira consulta de pré-natal, preconiza-se reconhecer os fatores de risco para TVP, a fim de realizar a estratificação do risco, o acompanhamento da paciente durante a gestação, e avaliar a melhor forma de prevenção, que pode ser feita de forma mecânica e/ou farmacológica, evitando possíveis complicações, como embolia pulmonar (PANASSOL MRC, et al., 2020).

Em relação aos métodos mecânicos, meias compressivas ajustadas pelo tamanho, dispositivos neuromusculares de eletroestimulação e de compressão pneumática intermitente da panturrilha ou flowtrons são comumente indicados no pós-operatório como forma de prevenção da TVP. Tais dispositivos aumentam o fluxo sanguíneo femoral por meio da compressão dos membros inferiores e estimulam a fibrinólise pelo aumento do fator ativador do plasminogênio tecidual e diminuição de seu inibidor. Para serem considerados eficazes, esses dispositivos devem ser usados continuamente, pois seus efeitos são reduzidos cerca de 10 minutos após sua remoção (UNGER HW, et al., 2018).

A tromboprofilaxia farmacológica por meio de anticoagulantes orais, como a varfarina, é contraindicada durante a gestação devido ao risco de anomalia congênita, porém é segura durante a amamentação. Em contrapartida, heparinas de baixo peso molecular (HBPM) não atravessam a barreira placentária e são associadas ao menor risco de eventos hemorrágicos materno-fetais, ausência de relatos de teratogenicidade, além da vantagem de não exigir monitoramento. No entanto, seu uso deve ser criterioso ou evitado em paciente com distúrbios hemorrágicos, acidente vascular cerebral hemorrágico, grave insuficiência renal, doença hepática grave, sangramento pré-natal e pós-parto ativo e risco de hemorragia grave (UNGER HW, et al., 2018).

A tomada de decisão sobre qual terapia adotar e sua duração depende do risco que a puérpera apresenta. Em pacientes com fatores de risco, como idade superior a 35 anos, índice de massa corporal maior que 30, imobilização por mais de 4 horas, varizes maciças, multiparidade e cesária de emergência, são indicadas meias de compressão associadas à HBPM, como enoxarapina ou deltaparina SC (SARNO MAC, et al., 2016). $\mathrm{Na}$ ausência de fatores de risco, são recomendadas apenas meias de compressão e deambulação precoce como tromboprofilaxia (EVANGELISTA MS, et al., 2018).

Em relação à duração da terapia, a tromboprofilaxia deve ser mantida por seis semanas em mulheres de alto risco e por dez dias em casos de risco intermediário. Em mulheres com fatores de risco persistentes, ou seja, com duração superior a dez dias pós-parto, como imobilização prolongada, infecção de ferida ou cirurgia durante o puerpério, a tromboprofilaxia deve ser prolongada até que o risco seja eliminado. Nessas puérperas, após 5 a 7 dias do parto, pode-se indicar a substituição do HBPM pela varfarina $5 \mathrm{mg} / \mathrm{dia}$ ou outro anticoagulante oral que não seja excretado pelo leite materno. Para isso, deve-se manter o uso da heparina associada à varfarina até que a razão entre o tempo de protrombina do paciente e o tempo de protrombina normal médio (RNI) atinja o nível terapêutico (2 ou 3) e o mantenha por dois dias seguidos (SCARABELI IC e ESTEVES APVS, 2020).

\section{CONSIDERAÇÕES FINAIS}

A partir dos dados acima, é possível concluir que o tromboembolismo venoso é mais recorrente nas pacientes durante o puerpério do que na gravidez. Desse modo, faz-se importante a estratificação de riscos para TEV desde $01^{\circ}$ trimestre gestacional, associando-os com o acompanhamento profissional preventivo $\mathrm{e}$ periódico durante o ciclo gravídico-puerperal. Assim, sob supervisão multiprofissional, é possível realizar um diagnóstico precoce, comumente realizado por exames de imagem, tal como a flebografia, que é o padrão ouro para o diagnóstico de TEV. Mediante a confirmação do diagnóstico, é importante a prescrição de um tratamento específico para pacientes com fatores de riscos associados. Dessa forma, torna-se explícito a importância do diagnóstico precoce para a doença, visando um melhor prognóstico para essas pacientes. 


\section{REFERÊNCIAS}

1. ABE K, et al. Venous thromboembolism as a cause of severe maternal morbidity and mortality in the United States. Semin Perinatol. 2019; 43(4): 200-204.

2. BATES SM, et al. Guidance for the treatment and prevention of obstetric - associated venous thromboembolism. Journal of thrombosis and thrombolysis, 2016; 41: 92-128.

3. CLINTON CM, et al. Prevention of postpartum venous thromboembolism. Obstet Gynecol Surv, 2019 ; 74 (10): 601 606

4. EVANGELISTA MS, et al. Venous Thromboembolism and route of delivery - Review of the literature. Rev Bras Ginecol Obstet, 2018; 40:156-162.

5. GALAMBOSI PJ, et al. Incidence and risk factors of venous thromboembolism during postpartum period: a populationbased cohort-study. Acta Obstet Gynecol Scand, 2017; 96: 853-861.

6. HART C, et al. Prevention of venous thromboembolism during pregnancy and the puerperium with a special focus on women with hereditary thrombophilia or prior VTE - position paper of the working group in women's health of the society of thrombosis and haemostasis (GTH). Hamostaseologie, 2020; 40 (5): 572-590.

7. HILLMANN BR, et al. Trombose de veia renal no puerpério: relato de caso. Revista Brasileira de Ginecologia e Obstetrícia, 2015; 37: 593-597.

8. MOHAMMED AA, et al. Pregnancy and Venous Thromboembolism: Risk Factors, Trends, Management, and Mortality. Hindawi BioMed Research International, 2020; 2020: 1-6.

9. OLIVEIRA ALML, et al. Tromboembolismo venoso na mulher: novos desafios para uma velha doença. J Vasc Bras, $2020 ; 19$.

10. PANASSOL MRC, et al. Trombose venosa profunda no puerpério: projeto consulta de enfermagem no pré-natal e pós-parto (CEPP) 12 anos de atuação. Revista Extensão em Foco, 2020; 21: 71-82.

11. PRESTI C, et al. Trombose Venosa Profunda: Diagnóstico e Tratamento. SBACV, 2015; 35.

12. RAMOS ASMB, et al. Eventos tromboembólicos em mulheres grávidas e puérperas. Enciclopédia biosfera, $2018 ; 15$ (28): 1213.

13. SANCHES SMV, et al. Thromboprophylaxis during the pregnancy-puerperal cycle - literature review. Rev. Bras. Ginecol. Obstet., 2020; 42 (4): 218-227.

14. SARNO MAC, et al. Trombose venosa profunda na gestação e puerpério. Maternidade Climério de Oliveira, Universidade Federal da Bahia, 2016.

15. SCARABELI IC, ESTEVES APVS. Fenômenos trombolíticos: profilaxia durante o ciclo gravídico puerperal. Editora Unifeso, 2020; 3: 88-97.

16. SILVA IGL, et al. Estratificação de risco para tromboembolismo venoso em pacientes de um hospital público do distrito federal. Cogitare Enfermagem, 2019; 24.

17. SOUZA EO, et al. Fatores de risco relacionados a coagulopatias no período gestacional. Revista Eletrônica Acervo Científico, 2021; 21: e6597.

18. SOUZA IA, et al. Assistência pré-natal e puerperal e indicadores de gravidade no parto: um estudo sobre as informações disponíveis no cartão da gestante. Rev. Bras. Saúde Mater. Infant., 2019; 19 (4): 991-997.

19. STUBBS MJ, et al. Trombose venosa profunda. BMJ, 2018; 350: k351.

20. THURN L, et al. Postpartum blood transfusion and hemorrhage as independent risk factors for venous thromboembolism. Thrombosis Research, 2018; 165 (2018) 54-60.

21. UNGER HW, et al. Venous thromboembolism in pregnancy. Obstetrics, Gynaecology and Reproductive Medicine, 2018.

22. VOON HY, et al. Tromboprofilaxia pós-parto em uma coorte multirreligiosa: uma revisão retrospectiva das indicações e aceitação. Journal of Obstetrics and Gynecology, 2018; 38(4): 493-497.

23. ZHOU ZH, et al. Tromboembolismo Venoso pos-parto precoce: fatores de risco e índice preditivo. SAGE Journals, 2018; 25: 6 . 